polarization characteristics of the band at $4792 \AA$ (band $\mathrm{A}$ ) as compared to that at $4795 \AA$ (band $\mathrm{B}$ ) on the $a b$ face. Band $\mathrm{B}$ has anomalous-type polarization, whereas band $\mathrm{A}$ has normal polarization characteristics. The two types of bands are observed throughout the spectrum. This observation might either lead to assigning the lower energy band to an electronic-phonon transition or else to an electronic transition for molecules at different sites.

PMDR $^{9}$ (phosphorescence microwave double resonance) is used to measure the zero field parameters as detected optically using the emission at the wavelenghts of these two bands. If band $B$ is a phonon band, its zero field parameters should be the same as for band A. On the other hand, if band A and band B are emissions from different sites, then the corresponding zero field parameters determined from detecting the emission of the two bands should be different. The result shows ${ }^{10}$ that $D=0.009849_{2} \mathrm{~cm}^{-1}$ and $E=0.014170 \pm 0.000002 \mathrm{~cm}^{-1}$ when band A is used, but $D=0.09857_{2} \mathrm{~cm}^{-1}$ and $E=0.014343 \mathrm{~cm}^{-1}$ when band B is detected in the PMDR experiment. The difference between the zero field parameters is similar to the previously observed difference of the zero field parameters of one guest molecule in different host sites." These results strongly indicate that bands $\mathrm{A}$ and $\mathrm{B}$ result from molecules at two different sites.

The next question that can be raised is: How much difference is there between the disposition of molecules in Site $A$ and those in Site B in the durene crystal? The answer to this question would also answer the important question: How much does the guest molecule have to rotate inside durene before it shows anomalous polarization? The interconversion of one site to the other at low temperatures could only occur if the degree of rotation involved is rather small. The ratio of the intensity of band $B$ to that of $A$ (which represents the ratio of the number of molecules in Site $B$ to that in Site A) is found to increase from a value of 4 at $4.2^{\circ} \mathrm{K}$ to $\sim 9$ at $40^{\circ} \mathrm{K}$. Interconversion of sites at low temperatures has been demonstrated previously ${ }^{12}$ for phenanthrene in biphenyl.

The above observations indicate that the anomalous polarization of 8-chloroquinoline phosphorescence in durene can result from small rotation from the substitutional disposition in the lattice. The need for crystal field mixing might be unnecessary to explain the anomalous polarization but indeed cannot be completely eliminated.

The authors wish to acknowledge the support of the Office of Naval Research.

* This work is done in partial fulfillment of the $\mathrm{PhD}$ requirements at UCLA.

$\dagger$ Alfred P. Sloan fellow.

¥ Present address: Fresno State College, Fresno, Calif.

$\$$ Contribution No. 2478.

1 N. K. Chaudhuri and M. A. El-Sayed, J. Chem. Phys. 43, 1423 (1965)

${ }^{2}$ S. M. Ziegler and M. A. El-Sayed, J. Chem. Phys. 52, 3257 (1970).
${ }^{3}$ S. M. Ziegler and M. A. El-Sayed (unpublished).

${ }^{4}$ C. A. Hutchison, Record Chem. Progr., Kresge-Hooker Sci. Lib. 24, 105 (1963).

${ }^{5} \mathrm{~J}$. S. Vincent and A. H. Maki, J. Chem. Phys. 39, 3088 (1963).

${ }^{6}$ M. A. El-Sayed, J. Chem. Phys. 47, 2200 (1967).

7 A. Forman and A. Kwiram, J. Chem. Phys. 49, 4714 (1968). ${ }^{8}$ R. Clarke, R. M. Hochstrasser, and C. J. Marzzacco, J. Chem. Phys. 51, 5015 (1969).

9 (a) J. Schmidt and J. H. van der Waals, Chem. Phys. Letters 2, 640 (1968). (b) D. S. Tinti, M. A. El-Sayed, A. H. Maki, and C. B. Harris, Chem. Phys. Letters 3, 343 (1969).

${ }_{10}$ These $D$ and $E$ values are determined from the peak maxima of the zero field microwave spectrum. In order to obtain the exact values, the hyperfine structure should be resolved and analyzed.

${ }^{11}$ R. E. Gerkins and A. M. Winer, J. Chem. Phys. 47, 2504 (1967)

${ }^{12}$ R. M. Hochstrasser and G. J. Smal], J. Chem. Phys. 48, 3612 (1968)

\section{Intermolecular Vibrations in Liquid Secondary Chlorides}

\author{
A. V. R. WARRIER AND S. KRIMM \\ Harrison M. Randall Laboratory of Physics, University of Michigan, \\ Ann Arbor, Michigan 48104 \\ (Received 24 November 1969)
}

Strong and broad absorption bands observed in the $100-20 \mathrm{~cm}^{-1}$ region in the case of many liquids have been interpreted as being due to pure rotational or to translation-rotation motions in the liquid state. ${ }^{1-3}$ The same explanation has been given for the observed fine structure of the near-infrared vibration bands of polar liquids dissolved in inert solvents and of polar molecules trapped in inert gas matrices. ${ }^{4-6}$ Chantry and co-workers ${ }^{7,8}$ suggested that the single band observed below $100 \mathrm{~cm}^{-1}$ in the far-infrared spectra of many simple liquids is due to a pseudolattice oscillation of the molecules of the liquid. This was further supported by their observation of strong and sharp bands in the same region of the spectrum when the liquid was crystallized. Jakobsen and Brasch $^{9}$ made far-infrared measurements on thirteen liquids and interpreted the observed absorption band as being due to "...vibrations involving the bond joining molecules together in dipole-dipole complexes."

TABLE I. Low frequency absorption bands of secondary chlorides.

\begin{tabular}{ccc}
\hline \hline Molecule & \multicolumn{2}{c}{ Frequency $\left(\mathrm{cm}^{-1}\right)$} \\
\cline { 2 - 3 } & $\begin{array}{l}\text { Room } \\
\text { temp. }\end{array}$ & $\begin{array}{c}\text { Liquid } \mathrm{N}_{2} \\
\text { temp. }\end{array}$ \\
\hline 2-Chloropropane & $\ldots$. & 69.5 \\
2-Chlorobutane & 67.0 & 67.5 \\
3-Chlorohexane & 67.0 & 69.0 \\
Meso-2,4-dichloro- & 68.5 & 69.8 \\
pentane & & \\
\hline \hline
\end{tabular}


In the present investigation, we have measured the far-infrared spectra $\left(100-50 \mathrm{~cm}^{-1}\right)$ of the following four secondary chlorides using a Perkin-Elmer 301 spectrometer: 2-chloropropane, 2-chlorobutane, 3-chlorohexane, and meso-2,4-dichloropentane. Measurements were made both at room and at liquid nitrogen temperatures. The 2-chloropropane and 2-chlorobutane spectra were recorded in the vapor state also. The 2-chlorobutane spectrum was recorded in addition as a solution in paraffin oil at different concentrations.

In all the liquids studied, one band was observed near $67 \mathrm{~cm}^{-1}$, of about $10 \mathrm{~cm}^{-1}$ half-width, which did not shift appreciably or sharpen even at liquid nitrogen temperature (see Table I). This band disappeared in the vapors studied and also in the case of 2-chlorobutane in solution.

Various interpretations are possible for the appearance of this band, viz., that it is due to a rotational or a translation-rotation motion of a single molecule in the liquid state, that it is due to a pseudolattice oscillation, that it is due to an intramolecular low frequency mode, or that it is due to an intermolecular vibration which is specific to the particular system.

If this band were due to a rotational or a translationrotation motion in the liquid, one would expect the frequency of this band to be different for the four molecules studied since their masses, and hence the moments of inertia, are different. Also, one would expect the band to persist even in solution. Both of these predictions are contrary to observation.

The appearance of the band at the same position also rules out the possibility of this band being due to a pseudolattice oscillation, which would also be expected to vary with the mass of the molecule.

If the observed band were due to any intramolecular motion, then one would observe the band in the vapor as well as in solution. This is not the case. Further, normal coordinate calculations by Moore and Krimm ${ }^{10}$ do not indicate any band at such low frequencies. We therefore do not believe that this band originates from an intramolecular vibration.

These arguments lead us to suggest that the observed $67 \mathrm{~cm}^{-1}$ band in these liquids is due to some specific intermolecular vibration. The constancy of the frequency of this band suggests that the intermolecular interaction is only through specific atoms in all of these molecules and that the corresponding vibration involves only the motions of these atoms.

We have also studied the $\mathrm{C}-\mathrm{Cl}$ stretching frequency of 2-chlorobutane in paraffin oil at different concentrations. In the pure liquid, this mode occurs at 607 $\mathrm{cm}^{-1}$ for the $S_{\mathrm{HH}}$, at $627 \mathrm{~cm}^{-1}$ for the $S_{\mathrm{HH}^{\prime}}$, and at 670 $\mathrm{cm}^{-1}$ for the $S_{\mathrm{CH}}$ conformation." ${ }^{11}$ The corresponding modes for the vapor are observed at 617, 631, and 682 $\mathrm{cm}^{-1}$, respectively. We found from our dilution studies that the $\mathrm{C}-\mathrm{Cl}$ stretching frequency increases from the liquid phase value to the vapor phase value as the concentration of 2-chlorobutane in paraffin decreases.
These results are consistent with the disappearance, in dilute paraffin solution, of an interaction between molecules in the pure liquid which involves the $\mathrm{C}-\mathrm{Cl}$ bond. The rise in $\mathrm{C}-\mathrm{Cl}$ stretching frequency in going from pure liquid to solution is similar to that which is found for hydrogen bonded systems. ${ }^{12}$

We wish to suggest the existence in these secondary chlorides of a specific weak intermolecular interaction between molecules which involves the $\mathrm{C}-\mathrm{Cl}$ bond. This may be analogous to a $\mathrm{C}-\mathrm{Cl} \cdots \mathrm{H}-\mathrm{C}$ hydrogen bond. The $67-\mathrm{cm}^{-1}$ band would then correspond to the localized motion involved in the stretching of this bond. The frequency shift of this band in polyvinyl chloride on deuteration is consistent with such an assignment. ${ }^{13}$ The disruption of this interaction in the vapor or in dilute solution would of course cause this band to disappear and would also be expected to lead to a small increase in the $\mathrm{C}-\mathrm{Cl}$ stretching frequency.

This research was supported by a grant from the National Science Foundation. We are indebted to J. J. Shipman of The B. F. Goodrich Company for furnishing us with samples of the secondary chlorides.

${ }^{1}$ P. Datta and G. M. Barrow, J. Chem. Phys. 43, 2137 (1965); 48, 4662 (1968).

${ }^{2} \mathrm{~S}$. G. Kroon and J. Van der Elken, Chem. Phys. Letters 1, 285 (1967).

${ }^{3}$ H. Freidmann and S. Kimel, J. Chem. Phys. 44, 4359 (1966).

${ }^{4}$ J. Kwok and G. W. Robinson, J. Chem. Phys. 36, 3137 (1962).

${ }^{5}$ G. E. Ewing, J. Chem. Phys. 37, 2250 (1962).

${ }^{6}$ M. O. Bulanin, N. D. Orlova, and D. N. Shchepkin, Opt. Spektrosk. 19, 731 (1965) [Opt. Spectrosc. 19, 406 (1965)].

${ }^{7}$ G. W. Chantry and H. A. Gebbie, Nature 208, 378 (1965),

${ }^{8} \mathrm{G}$. W. Chantry, H. A. Gebbie, B. Lassier, and G. Wyllie, Nature 214, 163 (1967).

${ }^{9}$ R. J. Jakobsen and J. W. Brasch, J. Am. Chem. Soc. 86, 3571 (1964).

${ }^{10} \mathrm{~W} . \mathrm{H}$. Moore and S. Krimm (private communication).

${ }^{11}$ C. Opaskar and S. Krimm, Spectrochim. Acta 23A, 2261 (1967).

${ }^{12} \mathrm{G}$. C. Pimentel and A. L. McLellan, The Hydrogen Bond (W. H. Freeman and Co., San Francisco, Calif., 1960).

${ }^{13}$ A. V. R. Warrier and S. Krimm (unpublished).

\section{Comments}

\section{Electronegativity and the Structure of Radicals}

\author{
T. A. Claxton and N. A. Smith \\ Department of Chemistry, The University, \\ Leicester, LE1, 7RH, England
}

(Received 23 December 1969)

Recently, Pauling, ${ }^{1}$ in a discussion concerning the structure of $\mathrm{CX}_{3}$ type radicals, stressed the importance of the partial ionic character of the $\mathrm{C}-\mathrm{X}$ bonds, as 